\title{
Comparisons of microstructures and texture and mechanical properties of magnesium alloy fabricated by compound extrusion and direct extrusion
}

\author{
H.-J. Hu ${ }^{\mathrm{a}, \mathrm{b}, *}$, Y.-L. Ying ${ }^{\mathrm{a}}$, Z.-W. Ou ${ }^{\mathrm{b}}$, X.-Q. Wang \\ a Chongqing University of Technology, Chongqing 400050, China \\ b PLA Chongqing Logistics Engineering College, 401311, China \\ c The University of Alabama, Tuscaloosa, AL 35487, USA
}

\section{A R T I C L E I N F O}

\section{Keywords:}

Magnesium alloy

Compound extrusion

Grain refinement

Basal texture

\begin{abstract}
A B S T R A C T
In this study, microstructure evolution, textures and mechanical properties of AZ61 magnesium alloy were investigated by optical microscopy (OM), scanning electron microscopy (SEM), transmission electron microscopy (TEM), and tensile tests. The samples were processed by a new compound extrusion (CE) which combines direct extrusion (DE) and two steps of equal channel anger extrusion (ECAE). The results show that $\mathrm{CE}$ process can refine the microstructure more effectively than the DE process. The CE-fabricated samples have a weaker texture (0002), and a more fine and homogeneous microstructures, which attributes to the additional two steps of ECAE in CE process. In CE process, twin dynamic recrystallization and rotational dynamic recrystallization occurred, which enhances the refinement of the grains and weakening of the texture. In addition, the samples fabricated by $\mathrm{CE}$ process display a higher tensile properties (yield strength, tensile strength and elongation) with an excellent balance of strength and tensile ductility. Based on this study, severe plastic deformation (SPD) techniques combining conventional DE and two steps ECAE into a single process are feasibility to improve the mechanical properties of AZ61 Mg alloy.
\end{abstract}

\section{Introduction}

Magnesium (Mg) alloys have become the focus in the world due to their characterizations [1,2], especially the high strength to weight ratios comparing to other superalloys [3], such as stainless steel [4,5], titanium alloys [6] and nickel-based super alloys [7,8]. Currently, $\mathrm{Mg}$ alloys have been widely applied in various industries, such as transportation, communications, electronics and aerospace, with more than $90 \%$ of them are in as-cast state. In the future, the increasing demand for large-scale applications of wrought $\mathrm{Mg}$ alloy products will determine the development of $\mathrm{Mg}$ alloys. However, the deformation capacity and strength of the traditional direct extruded rods are rather poor. It is well known that the finer the grain of the material, the better the mechanical properties of it. Therefore, mechanical properties of most metals can be effectively improved by refinement of their microstructure by alloying, processing, heat treatment and so on.

Several methods have been developed to refine the grains in $\mathrm{Mg}$ alloys, which includes rolling, heat treatment, recrystallization, hot extrusion, and severe plastic deformation processes, such as torsion, reciprocating extrusion. In recent years, bulk nanostructure materials that processed by severe plastic deformation (SPD has attracted the growing interest of specialists in materials science. Using radial forward extrusion (RFE), Jamali et al. [3] produced large diameter fine-grained seamless tubes with superior properties from smaller cylindrical billets. $\mathrm{Li}$ et al. [4] extruded $\mathrm{Mg}-4 \mathrm{Zn}-0.5 \mathrm{Ca}$ alloys at $280{ }^{\circ} \mathrm{C}$ using different ultra-slow extrusion speeds, and found that both the volume fraction and average size of DRXed grains increase with increase of the extrusion speed. Chang et al. [5] fabricated an AZ31 Mg alloy sheet using one-pass asymmetric hot extrusion at a temperature of $673 \mathrm{~K}$, and revealed asymmetric hot extrusion effectively weakened the basal texture and improved the ductility at room temperature. In addition, Shahzad et al. [6] performed extrusion of the DC-cast alloy AZ80 with different extrusion ratios. Ohhashi et al. [7] studied microstructure and mechanical properties of quasicrystals dispersed Mg alloys prepared by warm extrusion of the mixed powder of $\mathrm{Mg}$ and $\mathrm{Zn}-\mathrm{Mg}-\mathrm{Zr}$. Pardis et al. [8] manufactured $\mathrm{Mg}$ alloy using cyclic expansion-extrusion (CEE), and reported that the CEE could impose large strain values per pass while maintain a homogeneous hardness distribution in the sample's cross section as well. Yang et al. $[9,10]$ processed $\mathrm{Mg}$ alloy sheets by conventional extrusion and differential speed extrusion, and revealed that the process could enforce the near-surface microstructure to exhibit more dynamically

\footnotetext{
* Corresponding author at: Chongqing University of Technology, Chongqing 400050, China.

E-mail address: hhj@cqut.edu.cn (H.-J. Hu).
} 
recrystallized grains with the c-axis tilted about $12^{\circ}$ toward the extrusion direction. The extrusion process of AZ31 Mg alloy also has been simulated [11]. Wang et al. [12-15] have investigated the microstructure evolution in alloys processed by cyclic extrusion compression, which includes AZ31, AZ6, AZ91, and the Mg-10Gd2Y-0.5Zr $\mathrm{Mg}$ alloy. $\mathrm{Lu}$ et al. [16,17] combined the finite-element analytic technique with the microscopic observation to investigate dual-directional extrusion of $\mathrm{Mg}$ alloy. However, serve plasticity deformation technologies are difficult to be industrialized right now due to the complicate, high-costs of the processes.

One of the most often used SPD techniques is equal channel angular extrusion (ECAE) which could produce homogeneous submicron or nanocrystalline microstructures without reducing the cross section of the billet. Many factors have influences on the microstructures, such as rotation of specimens between consecutive passes, number of passes through the die (the increase of total strain) and temperature of deformation. There are many studies have investigated the ECAE process and obtained fine-grained $\mathrm{Mg}$ alloy with high strength and plasticity. Janeček et al. [18] fabricated.

squeeze cast AZ31 $\mathrm{Mg}$ alloy at $200{ }^{\circ} \mathrm{C}$ using ECAE up to four passes following route $\mathrm{Bc}$. Matsubara et al. [19] processed as-cast $\mathrm{Mg}-9 \% \mathrm{Al}$ alloy using a new processing procedure which involves the sequential application of extrusion and equal-channel angular pressing. Yapici et al.[20] compared the texture evolution of four different hexagonal close packed (hcp) materials subjected to one pass ECAE, and the resulting textures demonstrated a common feature where the basal poles were aligned along the most highly elongated direction of the sheared grains. Zhang et al. [21] evaluated the anisotropic mechanical behavior and its correlation with the microstructure and texture evolution of ECAE-processed AZ31 Mg alloy. Sepahi-Boroujeni et al. [22] fabricated as-cast AZ80 Mg alloy through the expansion ECAE.

However, there still exits an unbridgeable gap between the laboratory scale processing of nanocrystalline material and industrialization of ECAE. The main obstacle to spread the application of ECAE is low productivity, because ECAE usually involves a large number of steps which is not easily to be industrialized as it is not a continuous process. Even though ECAE was developed in the early 1980s, further study is still needed before its industrialization.

Through EX-ECAP which includes direct extrusion and equal channel angle pressing could produce excellent superplastic ductility including the low temperature super plasticity and the high strain rate super plasticity [23,24], it usually includes more than 2 steps, which means the material has to endure intricate diversification of forming environments and this leads to a higher chance of to be oxidized for the $\mathrm{Mg}$ alloy. Considering wrought $\mathrm{Mg}$ alloy products produced by plastic processing, such as plates, rods and tubes, having excellent properties, a new severe plastic deformation method named compound extrusion (CE) has been developed, which combines traditional direct extrusion (DE) and two steps ECAE. As the number of ECAE passes in CE can be adjusted by modifying the die structures, so the $\mathrm{CE}$ process can fabricate samples with the desired number of ECAE passes without the need to remove them from the die until rods are extruded out.

The present study aims to explore the effects of CE process and DE process on the microstructures, texture, and the mechanical properties of AZ61Mg alloy in room temperature, respectively.

\section{Experimental procedures}

\subsection{Materials and manufacturing methods}

The commercial available AZ61 Mg alloy billets were used in this study with a nominal composition (mass fraction) of $\mathrm{Mg}-6.11 \mathrm{Al}-$ 1.09Zn-0.23Mn (wt\%). Fig. 1 is the schematic diagram of CE process illustrating the configuration of designed and manufactured CE die. The CE die includes DE with an extrusion ratio of 11.6 and two steps ECAE, in which the die channel angle between DE and two steps of
ECAE, and the die channel angle between the two steps ECAE are both $120^{\circ}$. The diameter of rods extruded by the CE process is $25 \mathrm{~mm}$.

There are five deformation zones in ES die, in which the upsetting zone and sizing zone are assembled as DE zone, and two successive ECAE steps include the first ECAE step and the second ECAE step. Experiments were conducted on a horizontal extrusion machine with an extrusion ability of $500 \mathrm{t}$ and a container diameter of $85 \mathrm{~mm}$. Ascast billets were machined into round billets with a diameter of $80 \mathrm{~mm}$.

The billets and dies have been heated by furnaces,and the temperatures of billets are higher than those of dies to avoid heat loss of billets,and which would cause the extrusion of billets difficultly. The temperatures of dies and billets have been measured by infrared thermometer.Table 1 lists the process parameters of CE process which have critical effects on the microstructure refinements and mechanical properties. The punch moved along extrusion direction(ED), as shown in Fig. 1.

\subsection{Microstructural characterization and mechanical properties tests}

All the samples were taken from the center of longitudinal sections at different positions, and prepared using standard metallographic procedures including mounting, grinding and polishing. To observe the microstructure, the polished specimens were etched with an acetic acid, which contains $2 \mathrm{ml}$ acetic acid, $1 \mathrm{~g}$ picric acid, $5 \mathrm{ml}$ water, and $20 \mathrm{ml}$ of alcohol. Scanning electron microscope (SEM) with the samples prepared by smoothing the surfaces of samples. Microstructure observations were realized by means of a transmission electron microscope (TEM) instrument,the mechanical and electrochemical methods are used to reduce thickness of specimens firstly,and the ion thinning method will reduce thickness of specimens to a few nanometers.

$\mathrm{X}$-ray diffraction (XRD) were used to qualitative analyses of macrotexture. Standard compression and tensile sample of $\varphi 8 \times 16 \mathrm{~mm}$ and $\delta 5$ tensile samples were prepared. The meaning of $\delta 5$ is that the length of sample are 5 times the diameter of the sample. The mechanical properties of the samples were carried out in electronic universal testing machine CMT5150. The compression and tensile failure tests were conducted at a speed of $1 \mathrm{~mm} / \mathrm{min}$.

\section{Results and discussion}

\subsection{As-casted and as-homogenized microstructures}

Fig. 2(a) and (b) are the original optical microstructures for as-cast without/ with homogenized billets, respectively. The as-cast billet has an estimated average grain size of more than $200 \mu \mathrm{m}$ with intermetallic compound in a shape of continual networks that distributed either among grain boundaries or in interior grains, as shown in Fig. 2a. Fig. $2 \mathrm{~b}$ shows the optical microstructure of the cast ingot of the alloy after homogenized heat treatment at $673 \mathrm{~K}$ for $16 \mathrm{~h}$. It can be seen that the microstructure is much more uniformity, and the grains have a significant increase in size with an average size of $300 \mu \mathrm{m}$. This means that grains could grow up via homogenization treatment. In addition, after homogenization treatment, the continual networks microstructures become discrete, and intermetallic compounds have been dissolved into the matrix of AZ61Mg alloy.

\subsection{Strain rates distribution for different deformation methods}

The predicted strain rates were used to reveal the deformation characteristics of billets during DE and CE process as they could provide quantitative insight into the deformation behavior of billets. The effective strain rates contours of billets are shown in Fig. 3. There is a big difference between the distribution of strain rates for DE and $\mathrm{CE}$ process of billets. Compared to the strain rates of DE-processed samples, CE-fabricated AZ61 Mg alloy samples show an obviously 


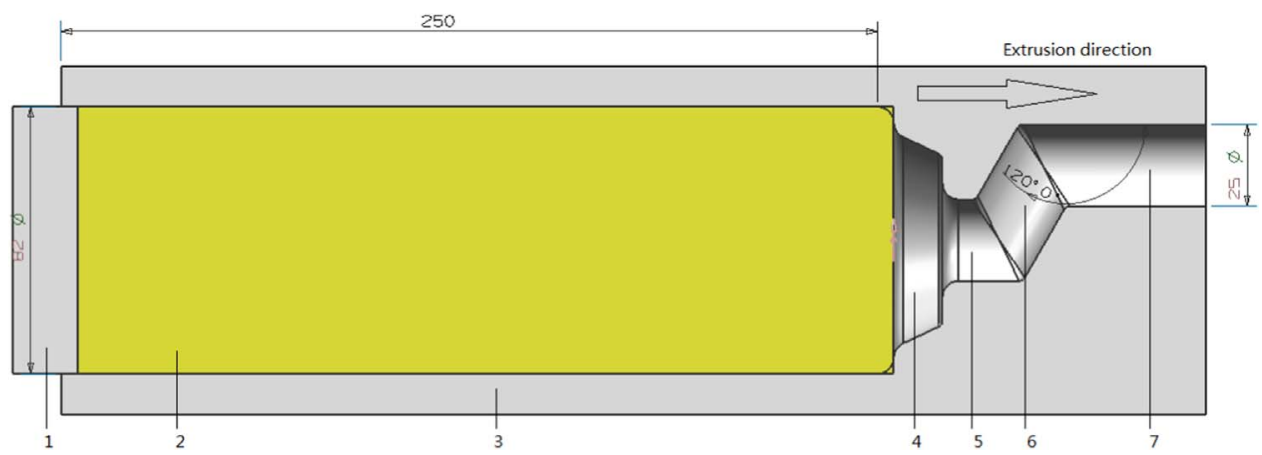

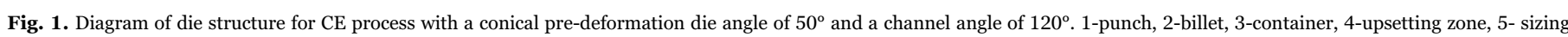
zone, 6-the first ECAE step, 7-the second ECAE step.

Table 1

Process parameters of CE process.

\begin{tabular}{llll}
\hline billet temperature & die temperature & container temperature & extrusion speed \\
\hline $400^{\circ} \mathrm{C}$ & $380{ }^{\circ} \mathrm{C}$ & $380^{\circ} \mathrm{C}$ & $0.5 \mathrm{~m} / \mathrm{min}$ \\
\hline
\end{tabular}

increased strain rates. The deformation of the initial extrusion is nonuniform. The highest strain of the deformation zone in Fig. 3a is located at positions of conical pre-deformation with a value of $20.4 \mathrm{~s}^{-1}$, while the maximum strain rate in Fig. 3b presents at the shear deformation of ED and two additional steps ECAE with a significant increased value of $74.2 \mathrm{~s}^{-1}$.

The principle of CE process [23] is to introduce compressive and accumulated shear strains into the samples, here, by DE and two steps shear deformation. The accumulative strain of $\mathrm{CE}$ process can be expressed as Eq. (1), which includes accumulative strain from DE (1st part) and two continuous ECAP steps (2ed part) [24,25].

$\varepsilon=\ln \lambda+2 *\left[\frac{2 \cot \left(\frac{\phi}{2}+\frac{\psi}{2}\right)+\psi \csc \left(\frac{\phi}{2}+\frac{\psi}{2}\right)}{\sqrt{3}}\right]$

where $\varepsilon$ is the accumulative strain, $\lambda$ is the extrusion ratio the inner corner angle, and $\Psi$ is the outer corner angle. Obviously, the effective strains in the rods decrease with rise of die channel angle $(\Phi)$ and the die outer corner angle $(\Psi)$. However, the die channel angle $(\Phi)$ has great influences on the effective strains in the rod. The strain rate of CE process can be expressed as Eq. (2).

$\dot{\varepsilon}_{2}=\frac{\varepsilon_{2}}{t^{\prime}}=\left[\frac{2 \cot \left(\frac{\phi}{2}+\frac{\psi}{2}\right)+\psi \csc \left(\frac{\phi}{2}+\frac{\psi}{2}\right)}{\sqrt{6}}\right] \frac{\sqrt{v_{2}}}{\psi R_{2}}$
According to Eqs. (1) and (2), preheated billets temperature might influence the extrusion speeds of extruded rods,and which would have some effects on the distributions of the strains and strain rates, but the parameters of $\mathrm{CE}$ die structures and extrusion speeds do have critical effects on them. Based on the results here, at the same extrusion ratios, the CE process could introduce a larger amount of shear deformation, strain rates, and Zener-Hollomon parameters in materials than DE process. The Zener-Hollomon parameter is known as the temperature compensated strain rate: $Z=e ́ \exp (\mathrm{Q} / \mathrm{RT})$,Where é is the strain rate, $\mathrm{Q}$ is the activation energy, $\mathrm{R}$ is the gas constant, $\mathrm{T}$ is the temperature.

\subsection{Optical microstructures}

Fig. 4 shows the optical microstructures of AZ61 Mg alloy processed by $\mathrm{DE}$ and CE process, respectively. Compared with the as-cast alloy in Fig. 3, the microstructure of DE-fabricated AZ31 Mg alloy sample in Fig. 4(a) has been refined obviously after extrusion. In addition, there were even equiaxed grains with an average grain size of $5 \mu \mathrm{m}$ in CEfabricated sample, which indicates that intensive dynamic recrystallization has taken place during $\mathrm{CE}$ process. This may attribute to the occurrence of dynamic recrystallization (DRX). It is characterized by fine DRXed grains and relatively coarse unDRXed grains. There is a relatively lower DRX volume fraction with the size of ultrafine DRXed grains over $20 \mu \mathrm{m}$ in Fig. 4a. For the CE process, the fraction of the dynamic recrystallization increases obviously simultaneously. After CE process, both the coarse grains $(\sim 100 \mu \mathrm{m})$ and fine grains have been effectively refined, around $10 \mu \mathrm{m}$, as shown in Fig. 4b. The homogeneity of the microstructure increases with rise of strain.

The Zener-Hollomon $(Z)$ reflects the degree of thermal deformation, and it is also important mechanical properties of the material parameters for hot deformation.

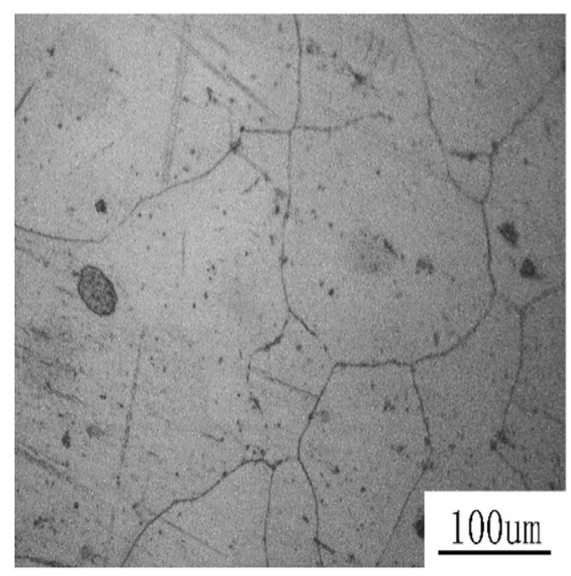

(b)

(a)
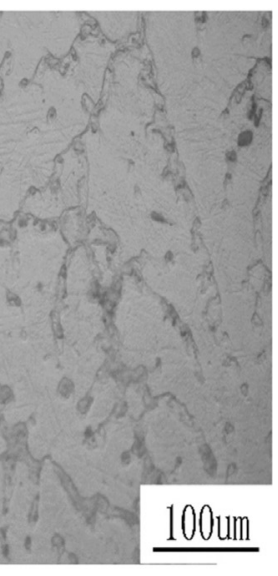

Fig. 2. Microstructures of initial AZ61 specimen (a) as-cast, and (b) homogenized at $673 \mathrm{~K}$ for $16 \mathrm{~h}$. 


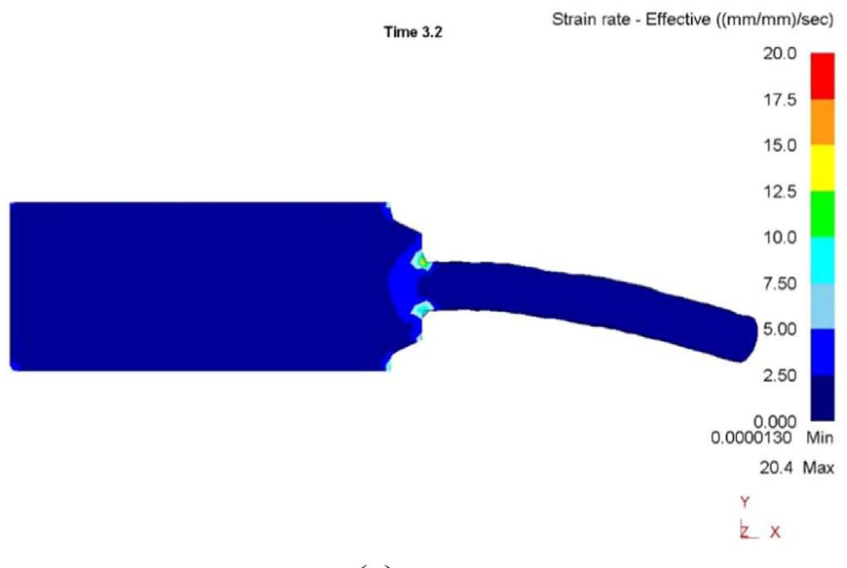

(a)

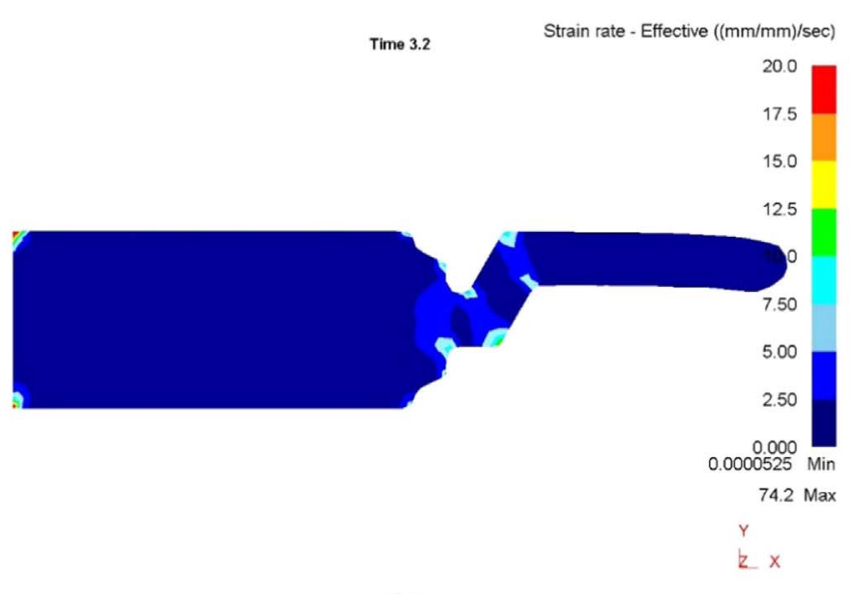

(b)

Fig. 3. The stress distributions of longitudinal section in rods at $400{ }^{\circ} \mathrm{C}$ for the $\mathrm{DE}$ process (a) and the CE process (b), respectively.

The Zener-Hollomon is a critical and effective parameter in the relationship between the average DRXed grain size (d) and the hot deformation parameters of temperature and strain rate $[25,26]$. It can be expressed by Eq. (3),

$-\ln (\mathrm{d})=A+\mathrm{B} \ln \mathrm{Z}$

where $d$ is diameter of grain size,which is measured by intercept
Table 2

Theory calculation results for dynamic recrystallization.

\begin{tabular}{lllllllll}
\hline$\varepsilon_{1}$ & $\varepsilon_{2}$ & $\varepsilon_{3}$ & $\dot{\varepsilon}_{1}$ & $\dot{\varepsilon}_{2}$ & $\dot{\varepsilon}_{2}$ & $\mathrm{Z}_{1}$ & $\mathrm{Z}_{2}$ & $\mathrm{Z}_{3}$ \\
\hline 2.42 & 3.15 & 3.81 & 1.77 & 0.72 & 0.78 & 1.74 & 0.84 & 0.86 \\
\hline
\end{tabular}

method with imageJ software, A and B are constants, and $\mathrm{Z}$ is the Zener-Hollomon parameter. For the first DE process, using $Z_{1}$ stands for the Zener-Hollomon parameter, then,

$Z_{1}=\frac{3 v_{1}}{R_{1}} \ln \lambda \exp \left(\frac{Q}{R T}\right)$

where $v_{1}$ is the extrusion speed. $\lambda$ is the extrusion ratio, and $R_{1}$ is the billet radius. Using $Z_{2}$ stands for the second phase for shearing,

$Z_{2}=\left[\frac{2 \cot \left(\frac{\phi}{2}+\frac{\psi}{2}\right)+\psi \csc \left(\frac{\phi}{2}+\frac{\psi}{2}\right)}{\sqrt{6}}\right] \frac{\sqrt{v_{2}}}{\psi R_{2}} \exp \left(\frac{Q}{R T}\right)$

where $v_{1}$ is the speed of extruded rods, and $R_{2}$ is the radius of extruded rod. Table 2 lists the theory results for the effective strains $(\varepsilon)$, strain rates $(\dot{\varepsilon})$, and Zener-Hollomon (Z) during the three stages of $\mathrm{CE}$ process, in which the subscript 1, 2, 3 stands for DE process, the first step of ECAE, and the second step of ECAE in CE process, respectively. Based on the results, it can be seen that large strains occurred during the CE process result in the fine grains.

It can be seen that the process and structure parameters of dies for the CE process have significant impacts on the dynamic recrystallizations of $\mathrm{Mg}$ alloy. The average size of the grains with high strains is finer than those with low strains. The high strains could inhibit the growth of the grains. So the size and volume fraction of dynamic recrystallizations of fined grains are inversely proportional to the accumulation strains [27,28].

In present study, the DRX occur easily with decreasing extrusion speeds at extrusion temperature as the critical stresses of DRX are strongly related to deformation temperatures and strain rates, and usually increase with the rise of strain rates at low temperatures. This is propitious to improve the nucleation rates and result in the refinements of DRXed grain size.

\subsection{TEM analysis of $M g$ alloy fabricated by $C E$ and DE process}

Fig. 5 presents two typical TEM micrographs showing the deformed microstructures after CE process. Unlike in the initial state of AZ61 Mg alloy, microstructures fabricated by CE process are inhomogeneous. Very fine particles were observed in the sample. New grains can be

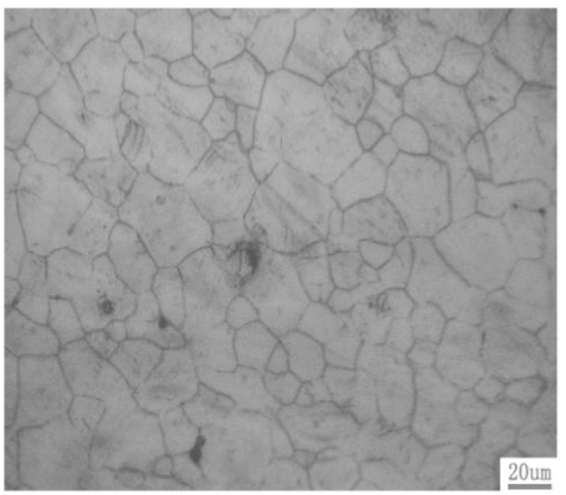

(a)

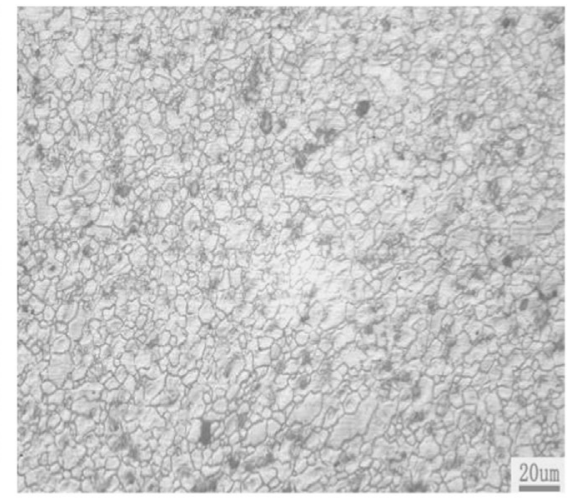

(b)

Fig. 4. Optical microstructures for AZ61 Mg alloy specimens fabricated by DE process (a) and CE process (b). 


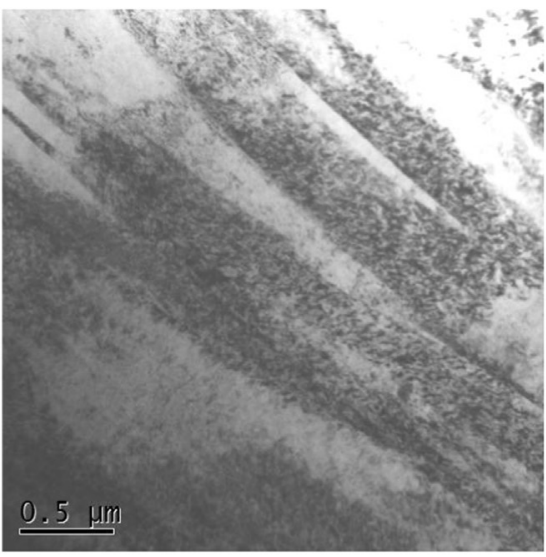

(a)

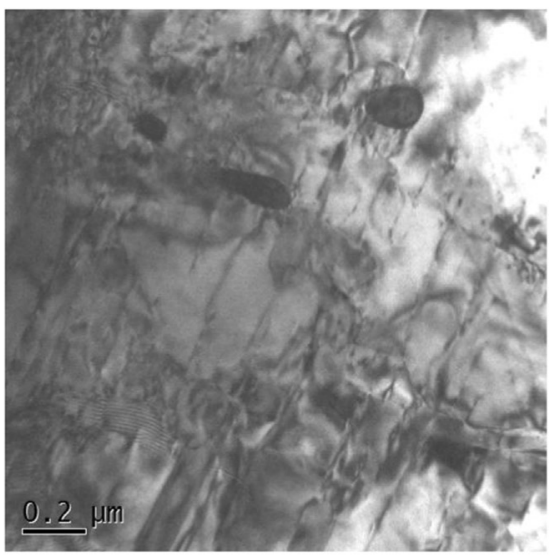

(b)

Fig. 5. TEM micrographs of twins (a) and dispersed distribution secondary phase particles (b) in the AZ61 Mg alloy fabricated by CE process.

generated by dynamic recrystallization and nucleation through bulging and subgrain rotation. Dynamic recrystallization behaviors of $\mathrm{Mg}$ alloys is coordinated with formations of secondary phases and twinning. Twinning deformation can play key roles in the deformation coordination of CE process. Parallel twins with narrow deformation bands cross over the whole transparent region of sample, and they are recognized as compressed twins as the width of them is about $100-500 \mathrm{~nm}$, as shown in Fig. 5a.

With continue of CE process, the first grain sharp boundaries and high misorientation angles appeared, and a non-uniform grain structure was observed, which is a mixed structure of coarse and fine particles. In addition, a lot of narrow deformation bands are formed in a certain angle with the extrusion direction (ED). The preferential nucleation of dynamic recrystallization occurred between these shear bands and the recrystallized grains have random orientations, which weakens the intensities of basal texture [29].

Therefore, the high strengthening results of $\mathrm{CE}$ process results in the appearance of dislocation density in the deformation twins and the dislocation tangles within deformation twins are very severe. Twin dynamic recrystallization (TDRX) is an important dynamic recrystallization mechanism of $\mathrm{Mg}$ alloy due to the higher storage capacity at the region of the twin compared to common grains. The TDRX process mainly contains the formation of dynamic recrystallization nucleation and the following deformation process. The grain boundary migration is converted to any grain boundary, and the crystalline grain grows up in the end.

Secondary phases shown in Fig. 5b may improve the dynamic recrystallization partially as they are progressively broken into rod-like or ball shape due to the large stress concentration in CE process. The average size of the large grains is about $400 \mathrm{~nm}$, while the mean size of smaller secondary particles is about $150 \mathrm{~nm}$, which means dislocations are just in some grains. The presence of dislocations in partial recrystallization grains may be attribute to the large strain imposed by CE process.

Mispairing of $\alpha-\mathrm{Mg}$ matrixes and secondary phase particles $\mathrm{Mg}_{17} \mathrm{Al}_{12}$ could increase the dislocation densities around the secondary phase particles, as shown in Fig. 6b. Higher dynamics of nucleation and growth may be imported into recrystallized grains. Continuous grain refinement in $\mathrm{CE}$ process could result in pinning force among boundaries of recrystallized grains. According to the above two aspects, grains size can be controlled and refined.

Two representative TEM photomicrographs of AZ61 Mg alloy fabricated by $\mathrm{DE}$ and $\mathrm{CE}$ process are shown in Fig. 6, respectively. Many of the grain boundaries for CE-fabricated AZ61 sample are uncertain with an indistinct appearance, which generally represents of high nonequilibrium grain boundaries. SAED pattern indicates diffracted spots-form rings which means there are high angles of misorientation for the grain boundaries.

\subsection{Texture of AZ61 Mg alloy fabricated by CE and DE process}

The polar diagram illustrates the crystal orientation (c-axis) orientation of the grains. Fig. 7 shows the (0002) pole figures taken from ED-TD plane of the samples by means of X-ray Diffraction (XRD). Fig. 7a shows that the largest pole densities of DE-processed sample concentrate on the center of (0002) pole figure. For most of the grains, basal plane is parallel to normal direction (ND) with a pronounced $\{0002\}$ basal texture, which was similar with conventional direct extrusion.

Fig. $7 \mathrm{~b}$ presents the (0002) pole figure for the CE-produced sample. The macro-texture of CE-prepared Mg alloy exhibits a good dispersity with a weak and spread of $\{0002\}$ planes toward transverse direction (TD). The maximum pole density of basal plane located in an angle away from ED with a certain deflection of normal direction (ND). The maximum intensity of the macro-texture is 2.915 for the CE-processed sample, which is significantly less than that of the DE-produced sample (9.686), as shown in Fig. 8a.

More recrystallized grains with the c-axis tilted toward the ED could be improved by the simple shearing introduced by two steps ECAE. The changed texture plays an important part in the improvement of mechanical properties of AZ61 Mg alloy. Compared to DE-processed samples, the effective strain could promote inclination of basal plane and weakens the basal texture intensity in CE-prepared sample.

In addition, three are a larger fraction of boundary having a misorientation angle over $30^{\circ}$ in the CE-processed sample than the DE-prepared specimen. This indicates that the CE process could increase the number of high angle grain boundaries (HAGBs) remarkably, which may results from the two additional shearings in $\mathrm{CE}$ process. In DE-processed sample, a large number of $\left\{\begin{array}{lll}10 & 1 & 2\end{array}\right\}$ twins appeared, while only a certain amount of secondary twins $\left\{\begin{array}{lll}10 & 1 & 3\end{array}\right\}-\{10$ 12
12 and $\left\{\begin{array}{lll}10 & 1 & 2\end{array}\right\}$ twins were generated in CE-processed specimen. Therefore, the number of tensile twins decreased significantly during the two steps of ECAE in CE process, and LAGBs can be transformed into HAGBs, which could refine the grains. Therefore, CE process can significantly refine the grain sizes, which indicates that the mechanical properties of $\mathrm{Mg}$ alloy could be improved. The recrystallization mechanism is continuous dynamic recrystallization.

Based on the former analysis, intensities of basal planes (0001) in deformed $\mathrm{Mg}$ alloys strongly depends on the deformation routes. In $\mathrm{CE}$ 


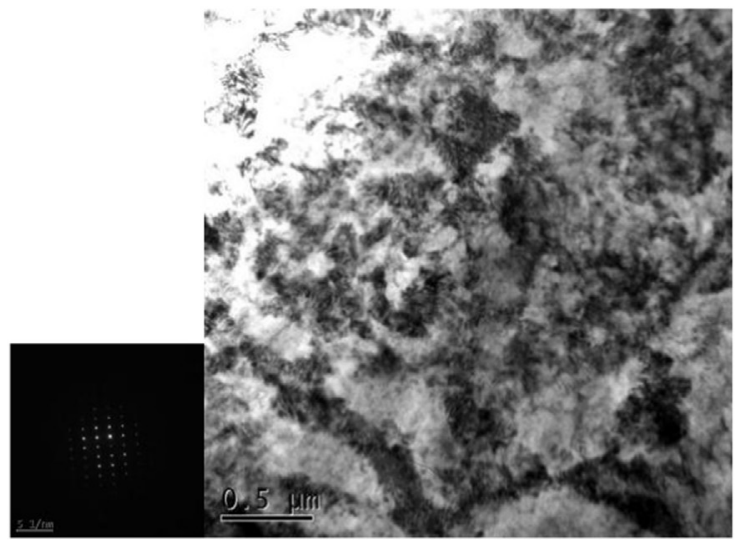

(a)

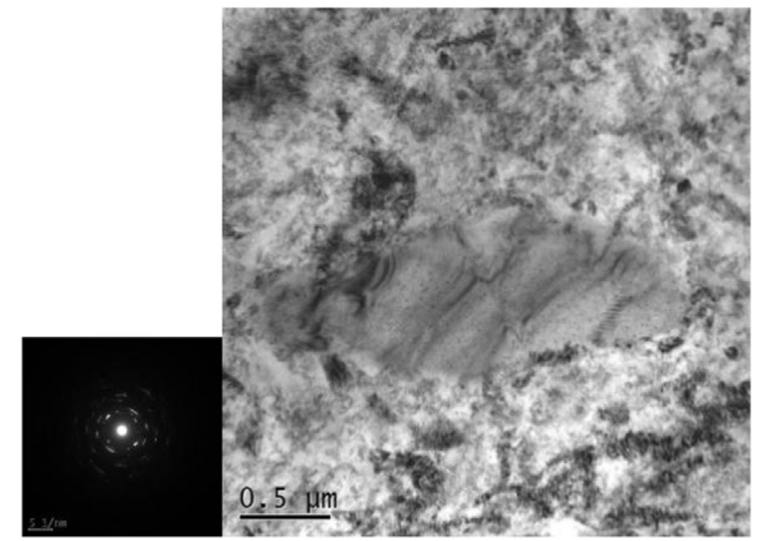

(b)

Fig. 6. TEM micrographs of the AZ61 Mg alloy fabricated by CE process (a) and DE process (b).
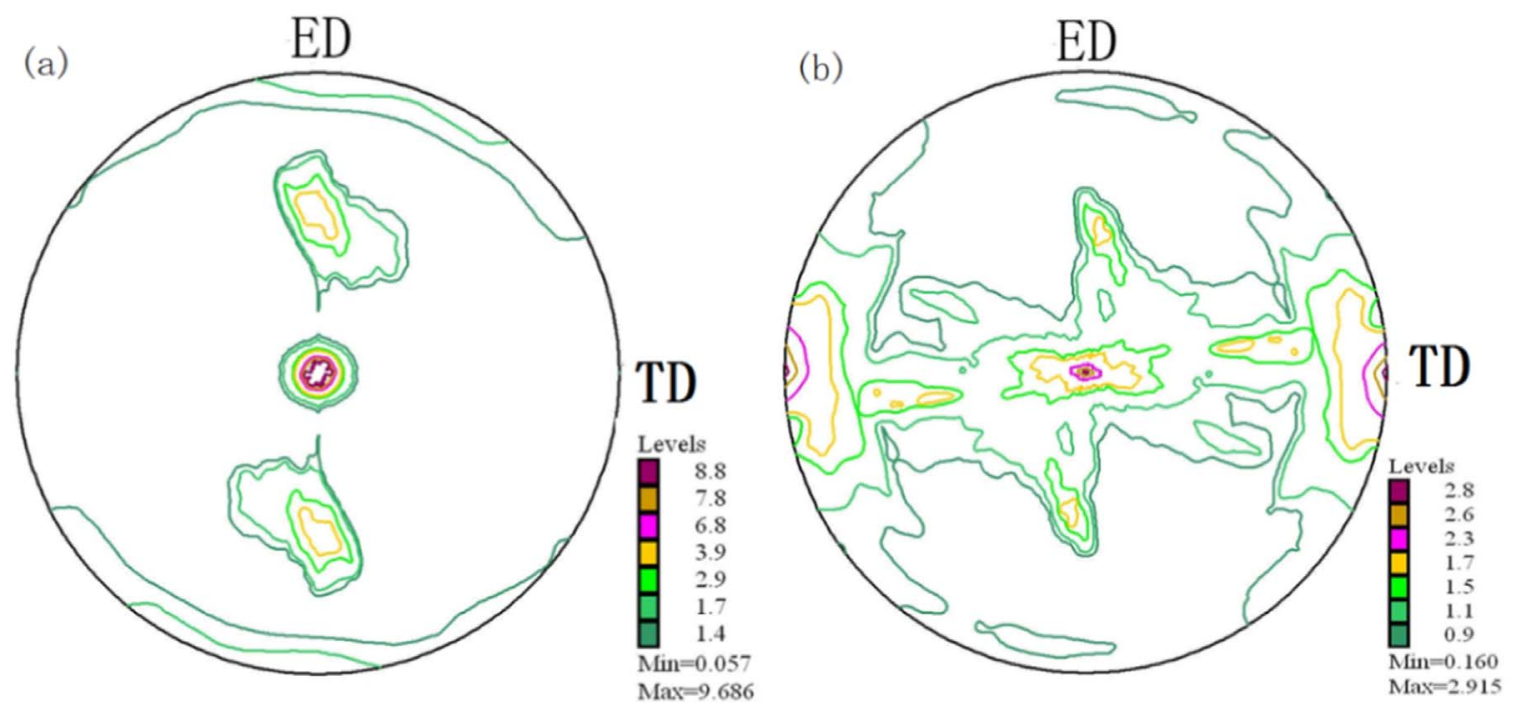

Fig. 7. The (0002) pole figures obtained from ED-TD plane of the sample fabricated by (a) DE process and (b) CE process by means of XRD.

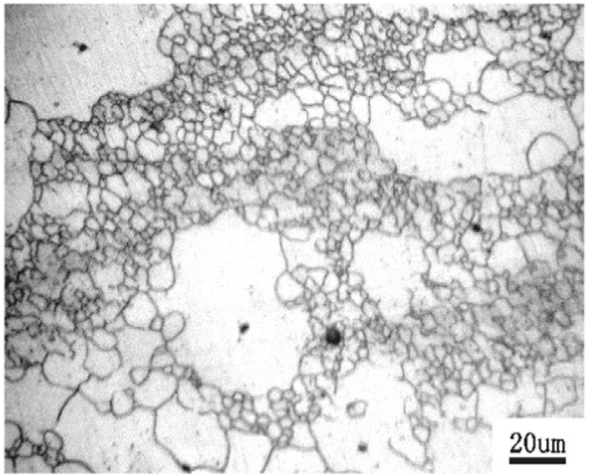

Fig. 8. Rotating dynamic recrystallization during CE process.

process, the basal plane (0001) is parallel to ED after DE zone, and in a certain angle to ED after the first ECAE step, then perpendicular to the ED after the second ECAE step. Grains may be rotated and slipped with development of plastic deformation, which may attribute to the $\{1012\}$ twin that is activated to accommodate the large angular rotations of the basal plane (0001) during the continuous deformation. Obviously, the continuous changing of (0001) orientation would weaken textures and introduce severe shear stresses process among grain boundaries and grains. Therefore, lattice twisted region appears at sufficient high stress

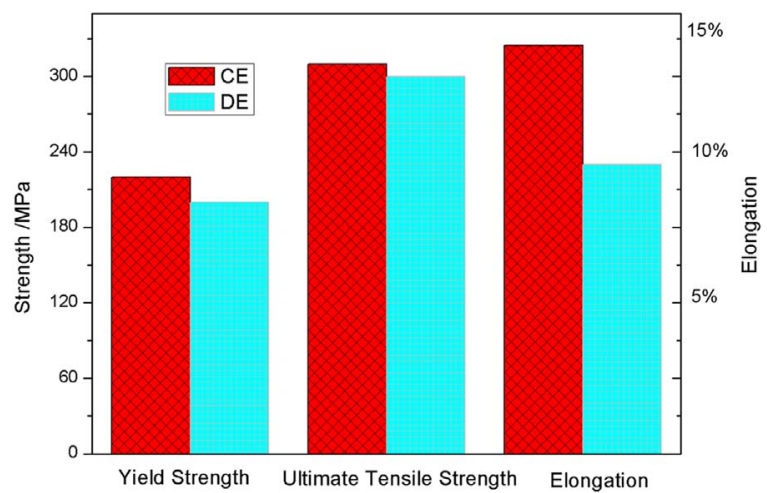

Fig. 9. Tensile properties of AZ61 Mg alloy samples fabricated by DE and CE process, respectively.

concentration zones with the boundaries between the coarse grains and the fined grains. It may occur both along grain boundaries and interior grains as ultrafine grains are easily to be deformed while coarse grains are more difficult to be deformed. Subgrains may appear in the severe deformation zones in the way of new grains with their orientations in favor of basal slip. The above mechanism could be considered as a kind of rotational dynamic recrystallization (RDRX), as shown in Fig. 8. 


\subsection{Comparisons of mechanical properties for AZ61 Mg alloy}

Tensile properties of AZ61 Mg alloy samples from tensile tests are shown in Fig. 9. In general, CE-prepared samples have higher tensile properties than DE-produced specimens with $9 \mathrm{MPa}$ higher in tensile strength, 9.5\% higher in yield strengths, $14.5 \%$ higher in elongation. This may attributes to the smaller average grain size in CE-fabricated samples as the smaller the average grain size, the higher the strength, which is based on Hall-Petch equation. That is because more grain boundaries in the component with a finer microstructure, which could hinder the movement of dislocations and this further leads to the harder and stronger comprehensive mechanical properties of the material. In addition, the tilted weak basal texture in the CE-processed samples also enhance the room temperature strength and plasticity. Therefore, severe plastic deformation (SPD) techniques which combines conventional DE and two steps ECAE into a single process are feasibility to improve the mechanical properties of AZ61 Mg alloy.

It is should be noted that the elongation of the CE-processed sample is expected to be larger than $14.5 \%$ as it contains finer grains. In addition, the elongation is also influenced by the internal stress, dislocation density, and metal forming process. Therefore, further study of the CE process is needed to improve plasticity of the $\mathrm{Mg}$ alloy based on the design idea of ES process combining DE and two steps of ECAE to increase the compression and shear stresses of the workpieces during the extrusion process.

\section{Conclusions}

The microstructures evolution of AZ61 Mg alloy during CE process and DE process have been investigated in the present study by optical microscope and transmission electron microscopy. Based on the experimental results, the major findings are listed as follow.

(1) Compared to DE-prepared samples, the effective strain, strain rates, and Zener-Hollomon parameter of CE-fabricated AZ61 Mg alloy samples increase significantly.

(2) CE process can refine the microstructure more effectively than the $\mathrm{DE}$ process. The CE-fabricated samples have a weaker texture (0002), and a more fine and homogeneous microstructures, which attributes to the additional two steps of ECAE in CE process.

(3) In CE process, twin dynamic recrystallization and rotational dynamic recrystallization occurred, which enhances the refinement of the grains and weakening of the texture.

(4) Comparing with DE-processed samples, the samples fabricated by CE process display a higher tensile properties (yield strength, tensile strength and elongation) with an excellent balance of strength and tensile ductility.

(5) Severe plastic deformation (SPD) techniques combining conven- tional DE and two steps ECAE into a single process are feasibility to improve the mechanical properties of AZ61 Mg alloy.

\section{Acknowledgements}

This research is funded by Chongqing Research Program of Basic Research and Frontier Technology (cstc2015jcyjBX0054), National Science Foundation of China (51101176 and 51571040), China Postdoctoral Science Foundation funded project (2015T81087 and 2014M552575). We would like to express our heartfelt gratitude to Professor Zhang dingfei and graduate student Geng qingmei, and they have instructed and helped me a lot in the past years.

\section{References}

[1] Q. Chen, Z. Zhao, G. Chen, B. Wang, J. Alloy. Compd. 632 (2015) 190-200.

[2] Q. Yang, B. Jiang, G. Zhou, J. Dai, F. Pan, Mater. Sci. Eng. A 590 (2014) 440-447.

[3] S. Jamali, G. Faraji, K. Abrinia, Mater. Sci. Eng. A 666 (2016) 176-183.

[4] Wei-jian Li, Kun-kun Deng, Xiao Zhang, Kai-bo Nie, Fang-jun Xu, Mater. Sci. Eng. A 677 (2016) 367-375.

[5] L.L. Chang, Y.N. Wang, X. Zhao, J.C. Huang, Mater. Sci. Eng. A 496 (2008) $512-516$.

[6] M. Shahzad, L. Wagner, Mater. Sci. Eng. A 506 (2009) 141-147.

[7] S. Ohhashi, A. Kato, I, M. D. P, Mater. Sci. Eng. A 528 (2011) 5871-5874.

[8] B. Talebanpour, R. Ebrahimi, S. Zomorodian, Mater. Sci. Eng. A 528 (2011) 7537-7540.

[9] Q. Yang, B. Jiang, J. He, B. Song, W.J. Liu, H. Dong, et al., Mater. Sci. Eng. A 612 (2014) 187-191.

[10] Q. Wang, B. Jiang, Y. Chai, B. Liu, S. Ma, J. Xu, et al., Mater. Sci. Eng. A 673 (2016) 606-615.

[11] S.J. Liang, Z.Y. Liu, E.D. Wang, Mater. Sci. Eng. A 499 (2009) 221-224.

[12] Q. Wang, Y. Chen, M. Liu, J. Lin, H.J. Roven, Mater. Sci. Eng. A 527 (2010) 2265-2273.

[13] T. Peng, Q.D. Wang, J.B. Lin, Mater. Sci. Eng. A 516 (2009) 23-30.

[14] Q. Wang, Y. Chen, M. Liu, J. Lin, H.J. Roven, Mater. Sci. Eng. A 527 (2010) 2265-2273.

[15] Y.J. Chen, Q.D. Wang, H.J. Roven, et al., J. Alloy. Compd. 462 (2008) 192-200.

[16] L. Lu, T. Liu, S. Jiang, F. Pan, Q. Liu, Z. Wang, Mater. Sci. Eng. A 527 (2010) 4050-4055.

[17] L. Lu, T. Liu, Y. Chen, et al., Mater. Trans. 51 (2010) 2088-2093.

[18] M. Janeček, M. Popov, M.G. Krieger, et al., Mater. Sci. Eng. A 462 (2007) 116-120.

[19] K. Matsubara, Y. Miyahara, Z. Horita, et al., Acta Mater. 51 (2003) 3073-3084.

[20] G.G. Yapici, I. Karaman, Mater. Sci. Eng. A 503 (2009) 78-81.

[21] D. Zhang, S. Li, Mater. Sci. Eng. A 528 (2011) 4982-4987.

[22] S. Sepahi-Boroujeni, F. Fereshteh-Saniee, Mater. Sci. Eng. A 636 (2015) 249-253.

[23] Kiyoshi Matsubara, Yuichi Miyahara, Zenji Horita, Terence G. Langdon, Metall. Mater. Trans. A. 35 (2004) 1734-1744.

[24] Dmitry Orlov, George Raab, Torbjorn T. Lamark, Mikhail Popov, uri Estrin, Acta Mater. 59 (2011) 375-385.

[25] Q. Chen, B. Yuan, J. Lin, X. Xia, Z. Zhao, D. Shu, J. Alloy. Compd. 584 (2014) $63-75$.

[26] Q. Chen, D. Shu, C. Hu, Z. Zhao, B. Yuan, Mater. Sci. Eng. A. 541 (2012) 98-104.

[27] Q. Chen, B. Yuan, G. Zhao, D. Shu, C. Hu, Z. Zhao, et al., Mater. Sci. Eng. A. 537 (2012) 25-38.

[28] R.H. Li, F.S. Pan, B. Jiang, H.W. Dong, Q.S. Yang, Mater. Sci. Eng. A562 (2013) 33-38.

[29] Q. Chen, Z. Zhao, Z. Zhao, C. Hu, D. Shu, J. Alloy. 509 (2011) 7303-7315. 